\title{
Correction to: Random Walk Exploration for Swarm Mapping
}

Miquel Kegeleirs (D), David Garzón Ramos (D), and Mauro Birattari (D)

\section{Correction to: \\ Chapter "Random Walk Exploration for Swarm Mapping" in: K. Althoefer et al. (Eds.): Towards Autonomous \\ Robotic Systems, LNAI 11650, https://doi.org/10.1007/978-3-030-25332-5_19}

In the metadata ("Cite this chapter as" section) of the originally published XML version and the author index of the volume the name of the second author was incorrectly stated as "Ramos, David Garzón". The name has been corrected to "Garzón Ramos, David". 